\title{
Blockchain Traceability to Ensure the Veracity of Diplomas
}

\author{
Khaled Mili \\ Quantitative Methods Department, College of Business Administration, King Faisal University, Al-Hasa, Eastern, Saudi Arabia \\ Email address: \\ kmili@kfu.edu.sa \\ To cite this article: \\ Khaled Mili. Blockchain Traceability to Ensure the Veracity of Diplomas. International Journal of Intelligent Information Systems. \\ Vol. 10, No. 4, 2021, pp. 60-68. doi: 10.11648/j.ijiis.20211004.14
}

Received: May 6, 2021; Accepted: June 16, 2021; Published: August 24, 2021

\begin{abstract}
Higher education institutions are considered one of the most important pillars and the greatest starting points from which the wheels of development and civilized advancement are launched, as well as their important role in instilling the values of society and preserving its moral and value system. In performing the role entrusted to it in achieving sustainable development and the comprehensive renaissance of society in various field and sectors. Thus; the accreditation systems are frequently used to verify which institutions are recognized and authorized to giveeducational or professional skills. However, these systems are not always efficient in countries where recognized higher education establishments cannot rally the demand for certified professionals required by the employment market. This generates a fertile argument for the "certificate factories" to sell false diplomas to unskilled people who are trying to catchbenefit of this deficit. In this regard, the digitization of diploma granting's and verification's processes, is becomingincreasingly important in order to guarantee the identity of diplomas, and that companies recruit the right qualified people. For that reason, an efficient management system for the control of diploma creation processes is immediatelymandatory. The Blockchain methodology provides efficient ways to examine the data information management systems. It is designed to make confidence techniques that can revolutionize information management methods. The major purpose of this paper is to develop a "BlockDipls" system based on Blockchain technology. This BlockDipls system is planned to support diploma traceability and smart contract functions, and can be used to address the problems of diploma falsification and diploma record fraud.
\end{abstract}

Keywords: Diploma Veracity, Blockchain, Traceability, Smart Contract

\section{Introduction}

Falsified diplomas have been a frustration on recruitment process since a long time. Indeed, human resources field beings have been searching for techniques to struggle various types of erroneous diplomas for years. Authentication is a cost-effectiveway of restriction, or even eradicating, falsified diplomas, and many frauds have been effectively controlled through diploma authentication. However, in 2015, a great scandal surrounding Carthage International Airport was reported in Tunisia and caused uproar among the population. As the first largest airport in Tunisia, the authorities found more than 200 employees working in the airporthad falsified university degrees [1]. More seriously, in 2017, the University of Kairouan in Tunisia, asked all establishments to verify the authenticity of documents of graduate students between 2012 and 2016 following a discovery of falsification of diplomas of achievement, diplomas of registration, report cards and diplomas in the university's administration [2]. In 2019 the Tunisian ministry ofhigh education and scientific research confirm that three of private universities were found to be in serious violation of the standards and Guidelines for Quality Assurance in Higher Education Area (SGQAH) [3]. These actions led to indignation among the Tunisian populace, and there has since been an urgent call to develop a credible anti-counterfeiting and traceability structure that ensuresdiplomas veracity and security in information management systems.

A number of universities all over the world have employed the blockchain technology to design different solutions and approaches related to higher education. The majority of solutions use the Bitcoin blockchain like Media Lab Learning Initiative [4]. A platform for creating, sharing, and verifying Blockchain-based educational certificates was proposed within the scope of the Digital Certificates Project. This incubation project is based on the Bitcoin blockchain and is lead by the Media Lab Learning Initiative at the 
Massachusetts Institute of Technology (MIT). This approach addressed the issues of digitizing academic certificates and does not investigate the possibility of the blockchain to be used in a globalhigher education credit and grading platform. The National University of La.

Plata (UNLP) has started developing a framework for a Blockchain based verification of academic achievement [5, 6], but no further details have been revealed until today [7]. The same approach was also adopted by the Argentinean College CESYT [7]. Both solutions use blockchain technology and cryptography (i.e. digital signature, time stamps, etc.) to issue diplomas for students. However, their approach does not address the issue of obtained credits for completed academic achievements. The approach is focused only on issuing diplomas (degrees) using the bitcoin blockchain. In 2016, the Parisian Leonardo da Vinci Engineering School (ESILV) announced it would certify diplomas on a bitcoin Blockchain [8]. They have partnered with the French Bitcoin startup Paymium, but no further details or a prototype have been published so far. There are also other higher education establishments, which have or intend to use the blockchain technology. In 2015, a software engineering school in San Francisco, the Holberton School, announced using the technology to help employers verifyacademic credentials [9]. Hence, the primary aim of this study is to address the question of falsified diplomas, and develop a blockchain-based structure, namely a "BlockDipls" to trace and manage the information in diploma supply chains. Additionally, the BlockDipls structure can provide valuable diploma recommendation information to different users. The study aims to extend theirsearch on information management systems by understanding how blockchain and machine learning technologies can be used in supply chain management inspired from Young et al. [10]. It is further expected that the findings will help other sup- ply chains address management issues and the challenges of safety supervision. Through this enquiry, the study attempts to concentrate on the following research questions: Question I: How can a structure be developed such that it manages establishments in the diploma creation process? Question II: How can blockchain technology be used to supervise the operations of the diploma creation process in management system? The paper is organized as follows. Section 1 presents an overview of the diploma's creation process; the key issues of using blockchain are discussed too. Section 2 presents related works. Section 3 provides the stylisme details behind the BlockDipls structure, in addition todetailing the smart contracts used for querying diploma records and detecting falsified diplomas. Section 4 reflects on the findings of the experimental results via simulation. The paper is concluded in Section 5 with a discussion on theneed for a comprehensive Blockchain stylisme methodology.

\section{Literature Review}

Ever-increasing numbers of researchers are realizing that Blockchaintechnology is not limited in its use to virtual currency transactions, but that it can also be applied to information management systems. For example, Li, Kang et al. [11] proposed an energy blockchain, a secure energy trading system using blockchain technology that addresses the security and privacy challenges caused by untrusted and non-transparent energy markets. Their results show that the energy blockchain is efficient within the industrial IoT (IIoT). Herbaut and Negru [12] studied a method of using blockchain-powered smart contracts and a network service chain to support a user-centric content delivery ecosystem. By presenting an example, in which blockchain facilitates machine-to-machine (M2M) interactions and establishing an M2M electricity market in the context of the chemical industry, Sikorski, Haughton, and Kraft [13] explored the applications of blockchain technology relating to the Fourth Industrial Revolution (Industry 4.0). Thakur, et al [14] explored using blockchain technology for land record management in India. The European Credit Transfer and Accumulation System (ECTS) [15] developed a learnercentered system to facilitate planning, delivery and evaluation of study programs and to facilitate student mobility by recognizing prior learning achievements, qualifications, experience and learningperiods. Learning results and the associated workload of a full-time academic year is assessed with 60 ECTS credits. Credits are expressed in whole num- bers. Considering the expected learning results and the estimated workload, different amounts of credits are assigned to different educational components (courses). Y. Qian et al. [16] confirmed that platforms for information sharingfor construction of smart cities are progressing but only between governmentagency and department. And they demonstrated that a blockchain-based approach enables effective information sharing with nontrusted organization and public during construction while preventing any illegal access and tampering of data. R. Zheng et al. [17] confirmed also that smart construction relies on BIM for manipulating information flow, data flow, and management flow but little efforts focusing on information security. They presented a blockchain- based architecture called "BcBIM" that can guarantee the data integrity and provenance. BcBIM can keep track of the last record modification without tampering. Chen, G., et al. [17] demonstrated that the blockchain technology can address trust issues and information sharing in construction industry. One of the methods is by integrating blockchain technology with building information modeling (BIM). Chen, G. et al. [18] presented in their paper the blockchain technology potential educational applications and explored how it can be used to solve some education problems. They confirm that the openness, borderless and permission less natures of blockchain technology can provide everyone equal access to the technology and the network built with it. Anyone can apply for an electronic wallet on blockchain network. Blockchain technology does not set any limits for the users. All schools, teachers, and students can apply it to daily, thus avoiding authority-bias. In this paper, and with the inspiration of B. Yong, et al. [10] and the ECTS as presented 
by EU Guide [15], we will introduce some studies of supply chain systems and we will use the blockchain technology in the diploma creation process.

\section{Stylisme of the BlockDiplos Structure}

Traditional and popular methods of diploma traceability and supervision are still based on the number labeling method, and there are three major problems involved: - First, numbers are easily fraudulent, which causes the hidden danger of forged records; - Second, the number system is too large to maintain on a national scale; - Third, diploma information is level to being corrupted with.

The blockchain can provide trustworthy data records for diploma traceability in the diploma creation process. When designing the BlockDipls structure, each diploma is assigned an $\mathrm{QR}$ code, which is used to input the diploma information conveniently and without much human workload. QR codes have been in usage since the early 2000's. Thesecuretechnology which is ableto solve two problems that exist in the number labeling method. For the third problem, that of tampered records, in theory, records in a general information system with a central server are easily corrupted with or deleted if the central server is attacked. However, the blockchain structure is a multi- center (or no-center) system, and it is difficult to attack all nodes, in order to corrupt with or edit records. Specifically, the BlockDipls structure focuses on three main establishments in the creation process, which are the ministry of high educationdesigned in this paper as the (measure deliver agency), the universities that provide the diplomas (providers), and the government (authorities). Records generated by these three establishments are permanently stored in the blockchain structure, and it is almost impossible to tamper with or edit the records. In fact, to tamper with a record, more than half of nodes must tamper with the record synchronously, which is very difficult for any university to achieve. The records in the blockchain structure are public and can be accessed by any intervenient for diploma traceability. As shown in figure 1, the BlockDipls includes three components, which are the SGQAH chain, the providers' chain, and the authenticity chain. These three chains are maintained by three establishments in the diploma supply chain, which are ministry of high education, the diplomas' providers (universities), and the authorities presented by the government. These three establishments use smart contracts to add or query diploma records. These smart contracts are expanded to the BlockDipls and executed on the Ethereum Virtual Machine (EVM) presented by I. Grishchenko et $a l$. in [19]. Guidance modules are described and tested to present instructions for usage, which includes a diploma demand forecasting module, a reliability evaluation measure, and an authenticity guidance measure. The diploma demand forecasting measure is implemented by Auto Regressive Integrated Moving Average (ARIMA) model and trained using history diploma demand data recorded in the blockchain. It can be used byrecruiters to control diploma conception within a reasonable range, in order to relieve the diploma dishonesty problem. The reliability evaluation measure is implemented by the ARIMA model and trained using diploma comments data stored in the blockchain. It can be used by the ministry of high education (measure deliver agency) to evaluate the reliability of providers. The authenticity recommendation measure is given to the authorities to inform users of the need for authenticate by using the historical authenticity records and fraud records stored in the blockchain.

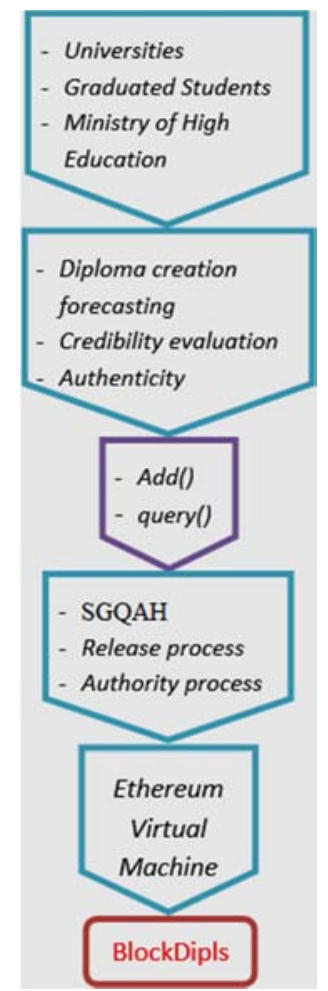

Figure 1. The architecture of the BlockDipls structure.

\section{The SGQAH chain}

Universities and all kind of establishments that provide diplomas put more social responsibility than ordinary establishments, where operation processes are subject to strict supervision by authorities. Their operation processes must conform to the stylisme in SGQAH standards, in order to record every progression in diploma creation. Standard Booklet of Conditions is a quality standard system for ensuring that results (diplomas) are made in a consistent manner. It is designed to minimize the risks involved in any creation that cannot be eliminated by testing the veracity of the diploma. As shown in (1) and (2) of Figure 2, SGQAH standards require establishments to record the creation in- formation of each diploma, including the name, the batch number, the creation date, and so on. In fact, even in many enterprises with SGQAH certification, quality management is still poor, and it is difficult to ensure the effective operation of internal audit, external audit, and management review. Therefore, in the stylisme of our BlockDipls, diploma-related records are recorded on the blockchain, including at least three types of record, which are the batch delivery record, the batch creation record, and the 
checkup record, as shown in figure 2.

The deliver process

The universities (providers) which deliver diplomas with SGQAH credentials can apply for delivery. The providers that decline the checkup or decline to approve the checkup, are not allowed to deliver their diplomas. The process of diploma delivery includes 07 steps: diploma creation, selfchecking, filling in the application, form printing, applying for release, collecting the diplomas and checkup, as shown in figure 3. The first five steps are conducted within universities, and the subsequent two steps (collecting and checkup) are carried out by the GA.

The first step is diploma creation, which should meet the SGQAH standards, as stated above. Then, the providers of the diplomas need to perform self- checking to ensure that their output is highly valuable and reliable. After selfchecking, the providers can apply for diploma from the in charge administration.

\begin{tabular}{|c|c|}
\hline (1)Batch delivery record & (2)Batch creation record \\
\hline $\begin{array}{l}\text { a- Diploma name, delivery form, batch } \\
\text { number, creation date } \\
\text { b- Date and time of delivery operation } \\
\text { c- Signature of person in charge of delivery } \\
\text { d- Signature of delivering operator } \\
\text { e- Inspection record according to process } \\
\text { regulations } \\
\text { f- Details of delivery operations, including } \\
\text { notebooks } \\
\text { g- Printed delivering materials with batch } \\
\text { numbers. } \\
\text { h- Abnormal record, investigation report and } \\
\text { signature } \\
\text { i- Check of delivering materials, delivering } \\
\text { diplomas. }\end{array}$ & $\begin{array}{l}\text { a- Diploma name and batch number } \\
\text { b- Date and time of creation } \\
\text { c- Signature of person in charge of each } \\
\text { creation process } \\
\text { d- Signature of reviewer } \\
\text { e- Batch number of each unprocessed and } \\
\text { supporting papers. } \\
\text { f- The number of parameters of major } \\
\text { creation equipment } \\
\text { g- Records of intermediate control results } \\
\text { and signature of operators } \\
\text { h- Abnormal record, investigation report and } \\
\text { signature }\end{array}$ \\
\hline (3) Inspection record & (4) Authenticity record \\
\hline $\begin{array}{l}\text { a- Diploma name, form, specification and } \\
\text { batch number } \\
\text { b- Quality standards and inspections } \\
\text { procedures } \\
\text { c- Type and number of instruments or } \\
\text { equipment used for inspection } \\
\text { d- Inspection process, including preparation } \\
\text { of reference solution } \\
\text { e- Inspection results, including observation, } \\
\text { calculation, curves... } \\
\text { f- Date of inspection } \\
\text { g- Signature of inspection } \\
\text { h- Signature of inspector and date of } \\
\text { inspection } \\
\text { i- Check the signature of the reviewer and } \\
\text { date of review. }\end{array}$ & $\begin{array}{l}\text { a- ID of diploma recipient } \\
\text { b- Gender of diploma beneficiary } \\
\text { c- Age of diploma beneficiary } \\
\text { d- Diploma name and batch number } \\
\text { e- Authenticity address } \\
\text { f- The date and time of Authenticity } \\
\text { g- Authenticity level } \\
\text { h- Authenticity department } \\
\text { i- Authenticity responsible }\end{array}$ \\
\hline
\end{tabular}

Figure 2. The batch delivery record, batch creation record, checkup record, and authenticity record of diplomas.

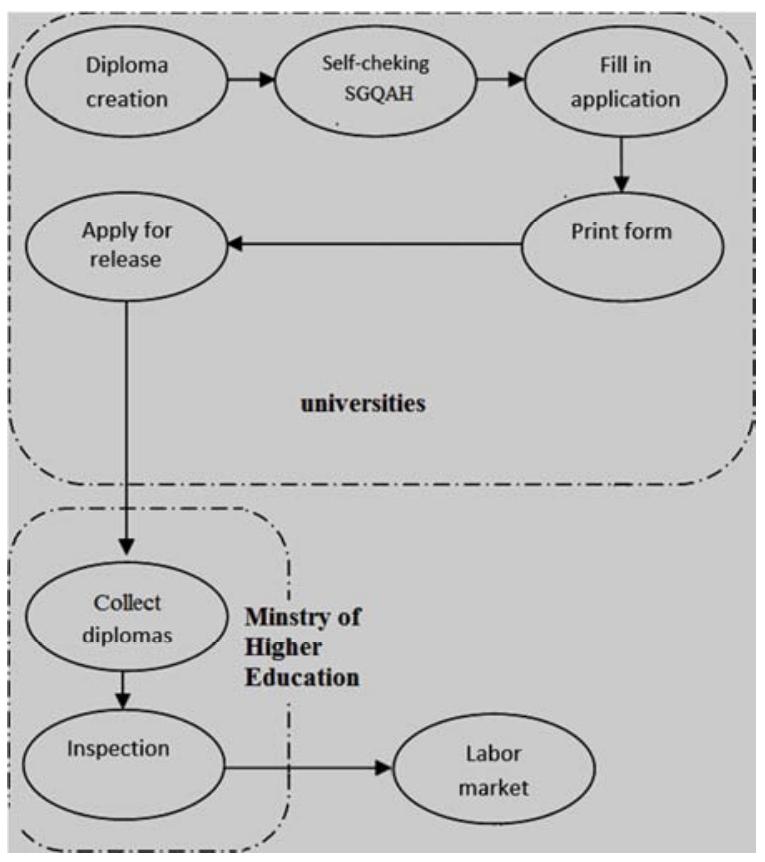

Figure 3. The process of diploma delivery.
Next, providers collect the proceeds of diplomas and fill in the application form. Then, the application form is printed. In the fifth step, the providers submit the application form and apply for measure deliver. After receiving the diplomas application, the authorities arranges spot diplomas in the administrative units of the providers. Then, the diplomas are sealed. Finally, if all the diplomas pass the checkup, the providers are given permission to deliver their diplomas into their graduated students. During the whole process, records of diploma creation submitted by providers are recorded on the SGQAH field. Checkup records, which are added by the measure deliver agency, should also be recorded on the Deliver process. After the measure deliver process, diplomas are sent to the authorities to be introduced to diploma beneficiary (students). When a student needs to be certified, the student submits an application to the authorities and then gets certified. Meanwhile, the accreditation record is submitted to the authenticity process.

The authenticity process

The authenticity process is used to store the accreditation records. The main information in this process is shown in figure 4, and includes basic information about diploma 
beneficiary, the authenticity department, and so on. In this, much personal information about diploma beneficiary is omitted to protect privacy. The identification of the diploma beneficiary is retained by the beneficiary and used to query personal authenticity information.

\section{Block structure information}

In traditional blockchain, a new block is generated by miners. In this paper, the primary architecture of Blockchain is neglected, where as an available structure, namely Ethereum, is adopted by our structure. Hence, only the block information will be discussed here. The block in the BlockDipls structure is used to store all diploma-related information, which is presented in figure 4 . In this paper, the idea is that each block contains three types of activities, which are transmitted by universities, the measure deliver agency, and the authorities.

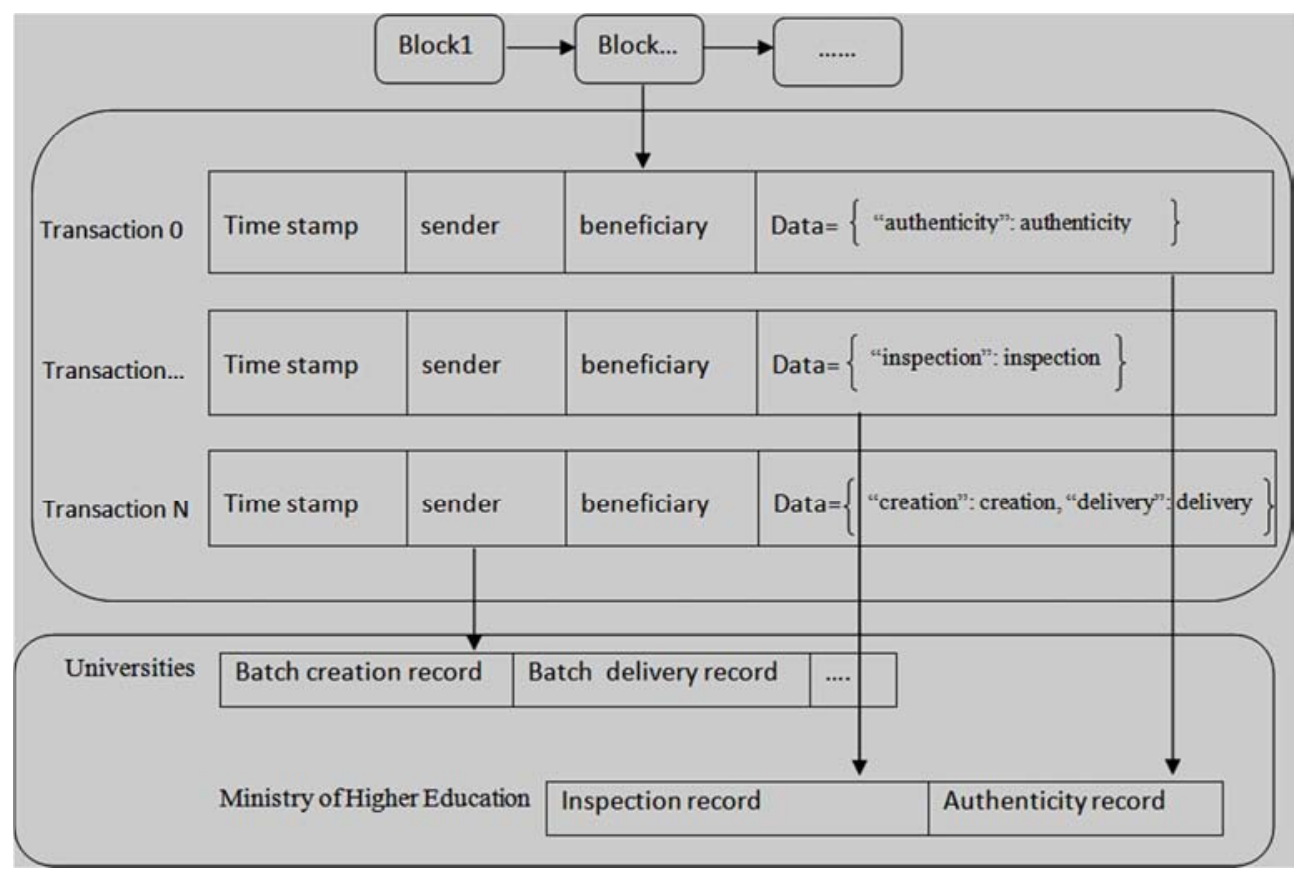

Figure 4. Structure information of the BlockDipls.

These activities are made up of four data fields, including a time stamp, the sender, the beneficiary, and data records content. The time stamp record the acitivity time. Sender represents the initiator of an activity, which is usually one of the three establishments. For the BlockDipls, the field of beneficiary is set as null, and the records are stored in the "data" field. The records are organized as a hash table structure, which is a dictionary-like collection of unique keys and their values. The keys of these hashs indicate the record class, such as "creation," "checkup," or "authenticity." The values of these hash tables exist in the form of an object, which contains record information and even other fixed items.

\section{Authority control}

In the Authoroty control procedure, if ministry of high education, the mea- sure deliver agency, or the universities want to upload their records, they must apply for accounts (including a public key and a private key) from the government. Additionally, the government maintains accounts for these establishments. The establishments upload records with a private key signature, and the signature is verified by the BlockDipls structure to ensure that the records are correctly submitted by the corresponding establishments. Accordingly, the government regularly checks records uploaded by these establishments. If there is any problem with the records, the corresponding establishments are investigated and potentially accused.

\section{Smart contracts for the detection of falsified diplomas}

In order to further realize the supervision function, and by designing suitable smart contracts defined by I. Grishchenko et al [19], our BlockDipls structure can intelligently monitor the status of the structure and provide query functions. Falsified diplomas events have been the cause of great social shock in Tunisia. By using the BlockDipls designed in this paper, along with and smart contracts, this problem can be controlled effectively. Before authenticity, the ministry of education needs to execute a detect() function invoked on the web middleware, as shown in figure 5. The detect() function queries the creation date to determine whether those diplomas are falsified. If the diplomas are falsified, the sendExpiration() function is invoked to send the information about the falsified diplomas to the blockchain structure, and all the institutions receive the information. Meanwhile, another authentic() function is not executed and the add() function is not used to add the authenticity record. Also, the structure does not execute the give() function (details in figure 7) to pay money to the authorities. In this way, the information about falsified diplomas is broadcast through the whole diploma supply chain as a reminder to the corresponding institutions, and the authenticity institution is not rewarded for forcibly introducing falsified diplomas. Furthermore, the universities providing falsified diplomas do not profit from it. 


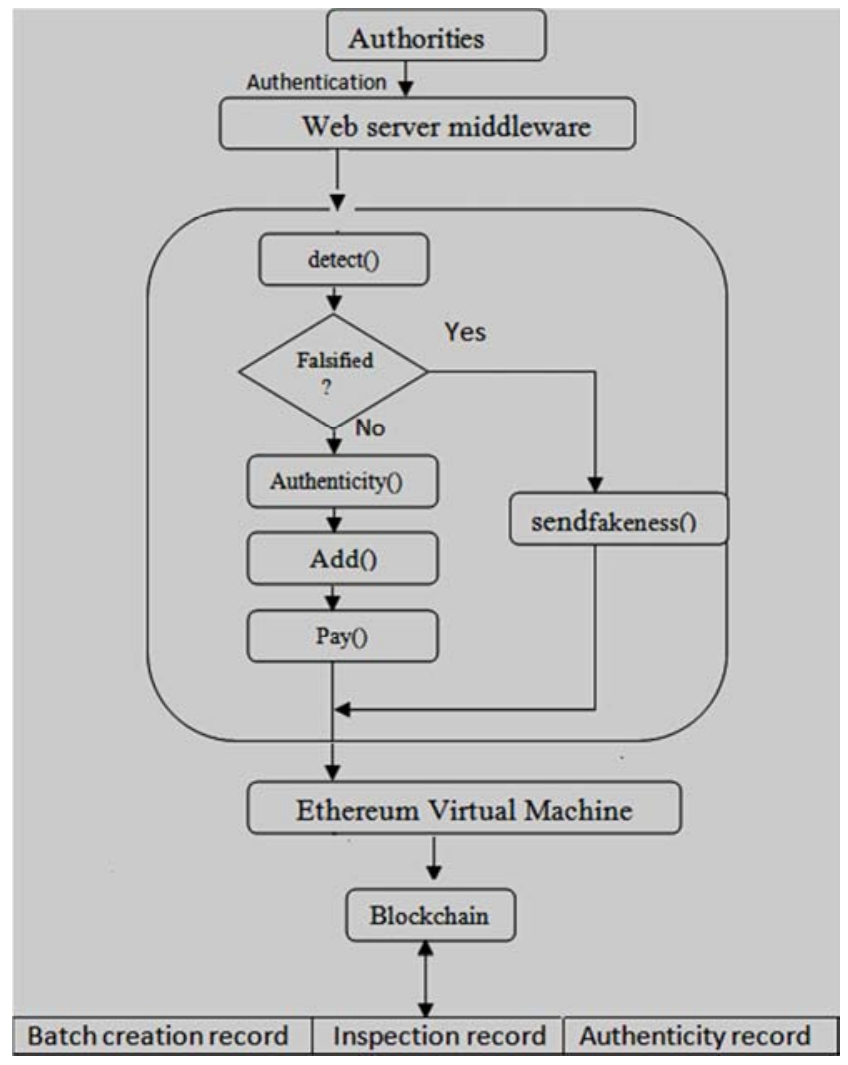

Figure 5. Flow of falsified diploma detection.

\section{Diploma information query}

Once a diploma problem occurs, the BlockDipls is used to trace the en- tire process of diploma circulation to investigate and affix responsibility for the problem. Hence, the BlockDipls structure provides the diploma queryfunctions, which are also implemented by smart contracts, as shown in figure 6 . The smart contracts are launched by message calls provided by the Ethereum platform. The parameters of the query are stored in the data field of the message. Then, the structure judges the identity via the parameters. Generally, two types of diploma records can be queried: one type is the authenticity record, which is raised by diplomas beneficiaries; another type is the diploma circulation record, which can be made by all three diploma establishments (universities, the ministry of high education, and the government). The chart flow is detailed in figure 6. For personal authenticity record queries from graduated students, the blockchain structure appeals the query(ID) function, for which the input parameter ID is the user identification. This function traverses the authenticity process in the BlockDipls to find the information of the student, and it returns the results of all the user's authenticity records. For a diploma circulation query initiated by the establishments, the blockchain structure executes another query diploma(batch-number) function, for which the input parameter batch-number is the batch number of the diploma. In this case, the function traverses the SGQAH process and the Deliver process to find the information of the diploma, and the query results are returned.

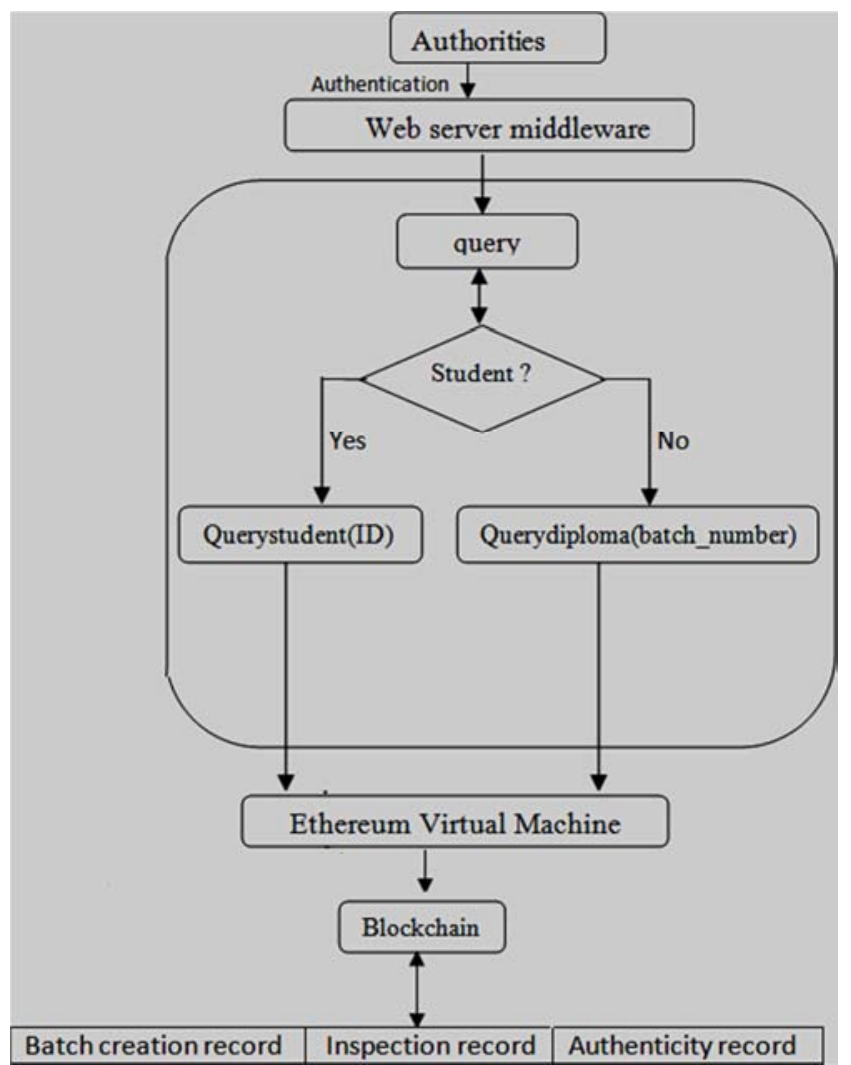

Figure 6. Flow of diploma information query.

\section{Diploma coin}

To exchange values along the BlockDipls creation process, a virtual currency is needed. The ERC20 token standard describes the functions and events that an Ethereum token contract needs to implement the functions and events inthe ERC20 standard as presented in the study of B. Young et al [10]. Traditionally, the students get theirdiplomas from universities in return to the graduations, and the authorities get diplomas from universities in return to the accreditation. These transactions are approved between students and universities, and universities and authorities (ministry of high education). With the ERC20 interface, we can implement a structure to transfer a token balance. In our stylisme, the diploma coin (DiploCoin) is concerned to replace these transactions between universities and the ministry of high education, and between the universities and their students. The DiploCoin is always specified as having a transfer ratio of 1:1 with legal tender. Therefore, the evaluation of DiploCoin is equal to the evaluation of officially certified standardize. As shown in figure 7, students (diploma beneficiaries) first $\log$ in to the web middleware and acquire their DiploCoins. After a successful graduation, the BlockDipls structure calls the give() function to finish the transaction. The give () function first calls the transferFrom() function to transfer DiploCoins from the address of the students to the address of the universities, with the total of DiploCoins being the present evaluation of the diploma (say, 200 DiploCoins). Next, the transferFrom() function is called once again to transfer DiploCoins from the address of the universities to the address of the authorities. This time, the 
total of DiploCoins is the exact judgment to the re- turns of the universities and the ministry of high education (200 DiploCoins). In fact, the diploma coin mechanism can be used effectively to supervise diploma veracity. The problematic or falsified diplomas will not increase returns, which will insist on these universities to give authentic diplomas.

\section{The Evaluation Framework}

Blockchain is most effective in dealing with a multistakeholder situation. A typical example of this type of environment is roaming services for mobile net- work operators (MNOs), where all the users rely on data and financial clearing houses (DCH/FCH) for the data, voice and financial reconciliation service. In our case, three entities are involved (authorities, students and universities). The authorities presented by the ministry of high education validate the transactionrecord. Blockchain provides a solution by introducing the authorities' party in which all accounting entries/transactions are cryptographically sealed by it and reside in a shared ledger. This third entry serves as a digitally signed receipt for the other parties involved in the transaction (students and universities). The success of blockchain implementation is never about the technology, but rather a new way of managing transactions cited by L. Jiang [20].

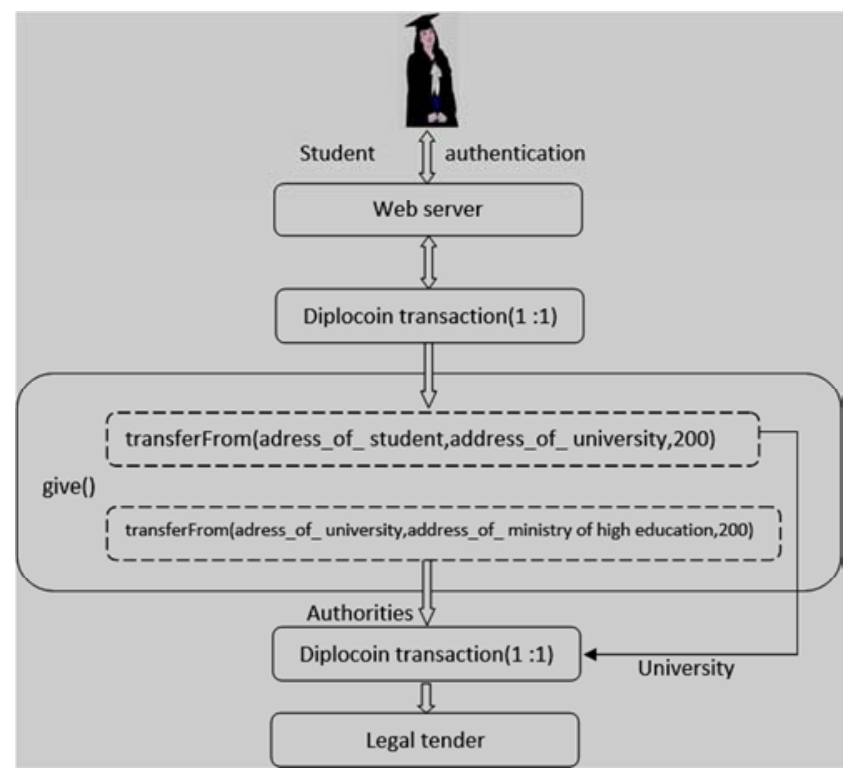

Figure 7. Flow of authenticity transactions by DiploCoin.

\section{Diploma demand forecast module}

One of the main causes of diploma falsification is the improper estimation of diploma demand. Therefore, a model that can accurately predict diploma demand is important for diploma creation process. The records in the BlockDipls structure can provide the total number of graduated students annually, which can be used to forecast diploma demand for the next year. Using the dataset for diploma demand forecast includes the number of diplomas in Tunisia from 1990 to 2014 [21]. The fitted ARIMA $(4,0,12)$ model is generated, and the finalforecast results in sample and out of sample are shown in figure 8 and figure 9 , respectively.

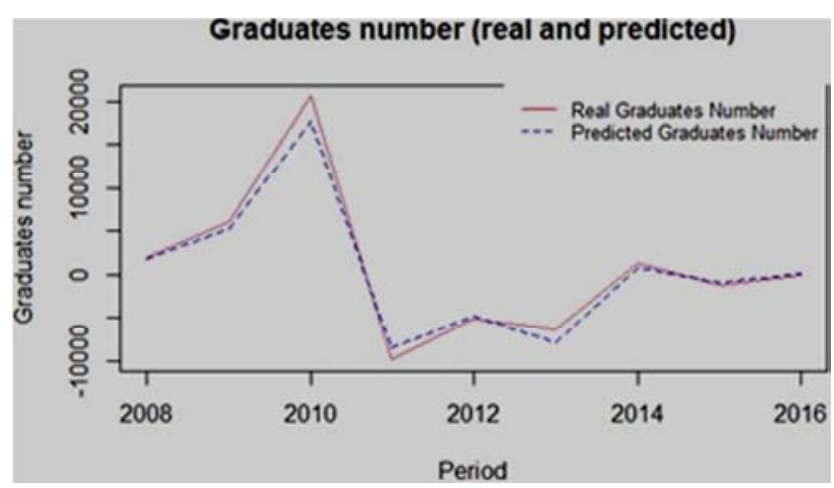

Figure 8. The diploma demand forecast from the ARIMA model.

We can notice that the forecasted diploma demand has approximately a similar trend to the real diplomas. By using the ShapiroWilk normality test, the results shown in figure 10 confirm that $\mathrm{W}=0.95108$, $\mathrm{p}$-value $=0.7019$, which indicates that diploma demand can be well forecasted. The median (residuals) are 278.9432, the mad residuals are 885.3579 and the mean residuals are 419.9286.

\section{Reliability Study}

In the classical systems, there is a potential for a single point of failure due to the existence of a single and centralized third-party. However, our proposed solution in this paper achieves more resilience than the classical systems due to the decentralization and distributed characteristics of the blockchain, which confirms the innovation of our approach.

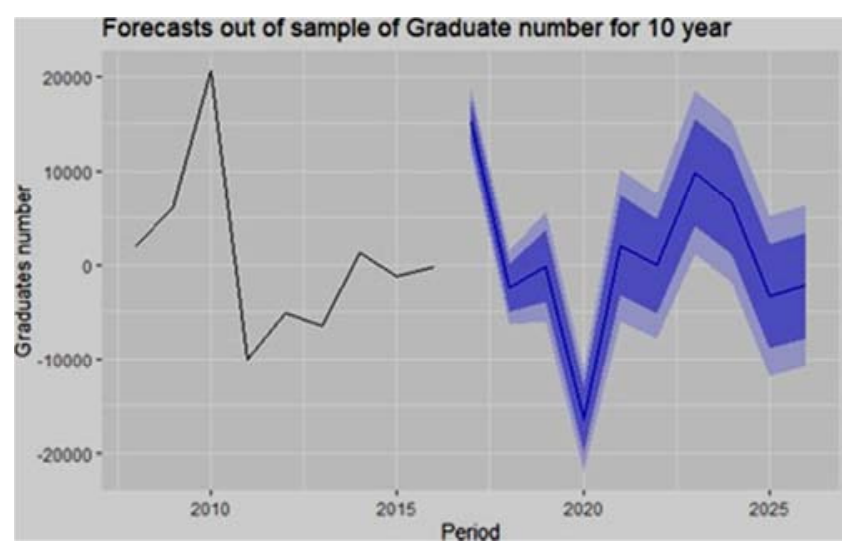

Figure 9. The ARIMA model for 10 years.

\section{Conclusion}

This paper examined the problematic of applying blockchain technology to diploma creation process. Meanwhile, smart contracts based on Ethereum for querying personal authenticity records and diploma flow were designed for students, universities and authorities, to trace diploma operation records. We demonstrated that the issue of diploma legitimate responsibility can be resolved using the BlockDipls structure. Additionally, we also designed smart contracts to 
detect falsified diplomas. In addition, a diploma demand forecasting is also introduced into the diploma system for evaluation functions. The experimental results showed that blockchain technology could ensure that diploma records are not corrupted with. Our study suggests that it will be of major benefit to use blockchain technology. Blockchain can be adopted to store reliable data records, which are exactly what authorities need to train reliable approval modules. Generally, this study indicates that the blockchain can help form an honest circle and improve creation process functions in supervising system. The process of diploma application and automated diploma yielding are deliver and transparent in our BlockDipls structure. Companies, organizations or any user can consequently find out for information on any diploma from the system. In conclusion, the structure assures information veracity and guarantee.

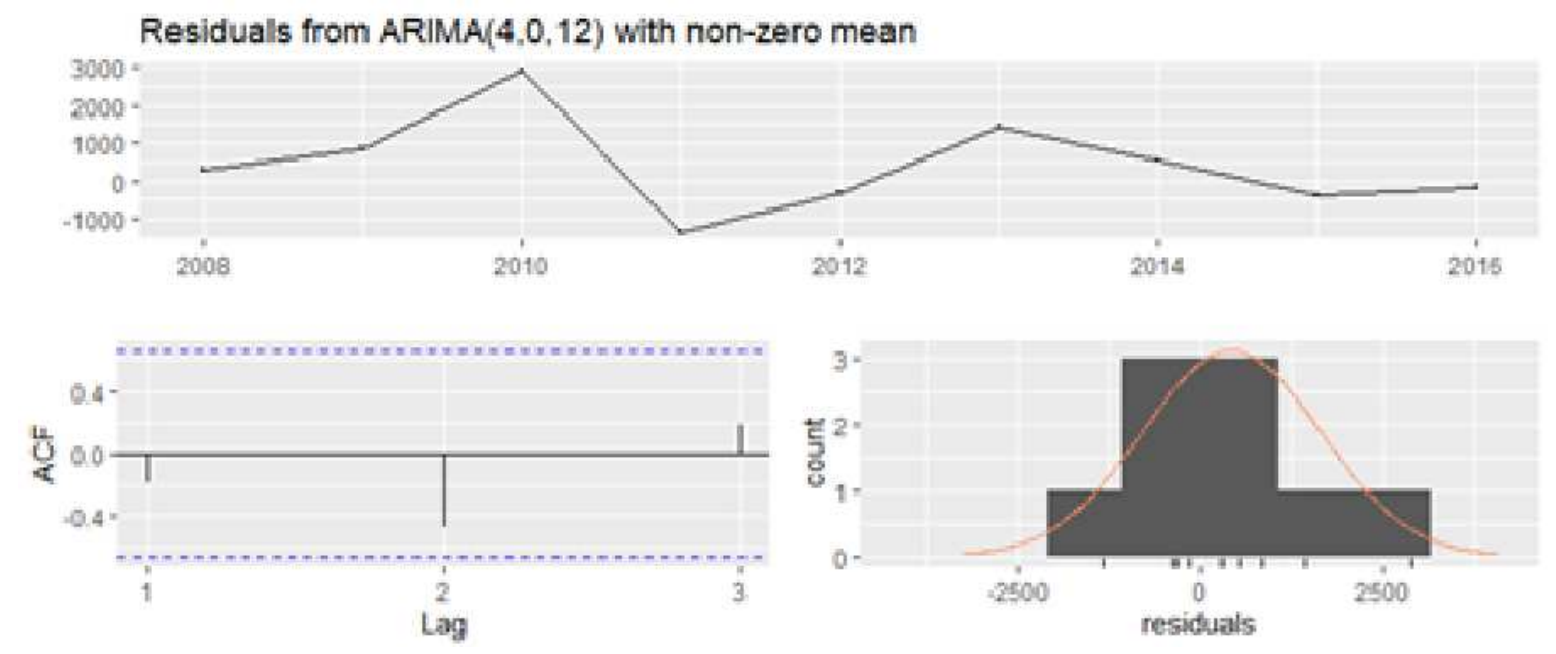

Figure 10. The Shapiro-Wilk normality test results.

\section{References}

[1] Wepost, Tunisair: 200 fonctionnaires avec des diplômesfalsifiés! (2017). URL http://www.wepostmag.com/tunisair-200fonctionnaires-diplomes-falsifies/

[2] Shemsfm, Falsificationav' er' eedesnotesetdes document sofficiels del' universit' e des lettreset des sciences humaines a regada (Apr 2017). https://www.shemsfm.net/fr/actualites_tunisie-news_newsregionales/165219/falsification-averee-des-notes-et-desdocuments-officiels-de-1-universite-des-lettres-et-dessciences-humaines-a-regada

[3] L. Presse, Fermetured' uneuniversit' epriv' eetaudit approfondide septautres institutions university airespriv'ees (oct 2019). https://lapresse.tn/32635/fermeture-duneuniversite-privee-et-audit-approfondi-de-sept-autresinstitutions-universitaires-privees/

[4] M. L. L. Initiative, Digital certificates project. URL https://learn.media.mit.edu/

[5] U. N. D. la Plata. [link]. URL https://unlp.edu.ar/

[6] F. Bond, F. Amati, G. Blousson, Blockchain academic verification use case (2015).

[7] F. Amati, First official career diplomas on bitcoin'sblockchain (2015). URL https://blog.signatura.co/_rst-of_cialcareerdiplomas-on-bitcoin-s-blockchain-69311 acb544 $\mathrm{d}$

[8] S. Das, Parisian engineering school will certify diplomas on the blockchain - cryptocoinsnews (2016). URL https://www.cryptocoinsnews.com/ parisian-engineeringschool-willcertify-diplomas-blockchain/
[9] L. Coleman, Engineering school simplifies verifying certificates using the blockchain-cryptocoinsnews; 2015 (2015). URLhttps://www.ccn.com/engineering-schoolsimplifies-verifying-certificates-using-bloc

[10] B. Yong, J. Shen, X. Liu, F. Li, H. Chen, Q. Zhou, An intelligent blockchain-based system for safe vaccine supply and supervision, Interna- tional Journal of Information Management (2019) 102024.

[11] Z. Li, J. Kang, R. Yu, D. Ye, Q. Deng, Y. Zhang, Consortiumblockchain for secure energy trading in industrial internet of things, IEEE transactions on industrial informatics 14 (8) (2017) 3690-3700.

[12] N. Herbaut, N. Negru, A model for collaborative blockchainbased video de- livery relying on advanced network services chains, IEEE Communications Magazine 55 (9) (2017) 70-76. doi: 10.1109/MCOM.2017.1700117.

[13] J. J. Sikorski, J. Haughton, M. Kraft, Blockchain technology in the chemical industry: Machine-to-machine electricity market, Applied Energy 195 (C) (2017) 234-246. doi: 10.1016/j.apenergy.2017.0.

https://ideas.repec.org/a/eee/appene/v195y2017icp234-246. html

[14] V. Thakur, M. Doja, Y. K. Dwivedi, T. Ahmad, G. Khadanga, Land records on blockchain for implementation of land titling in india, In- ternational Journal of Information Management $52 \quad$ (2020) $101940 . \quad$ doi: https://doi.org/10.1016/j.ijinfomgt.2019.04.013. URL https://www.sciencedirect.com/science/article/pii/ S0268401219303329

[15] E. U. Guide, Publications office of the europeancommision (2015). URL https://op.europa.eu/s/oNuY 
[16] Y. Qian, Z. Liu, J. Yang, Q. Wang, A method of exchanging data in smart city by blockchain, in: 2018 IEEE 20th International Conference on High Performance Computing and Communications; IEEE 16th International Conference on Smart City; IEEE 4th International Conference on Data Science and Systems (HPCC/SmartCity/DSS), 2018, pp. 1344-1349. doi: 10.1109/HPCC/SmartCity/DSS.2018.00223.

[17] R. Zheng, J. Jiang, X. Hao, W. Ren, F. Xiong, Y. Ren, Bcbim: A blockchain-based big data model for bim modification audit and prove- nance in mobile cloud, Mathematical Problems in Engineering 2019. doi: 10.1155/2019/5349538.

[18] G. Chen, B. Xu, M. Lu, N.-S. Chen, Exploring blockchain technology and its potential applications for education, Smart Learning Environments 5 (2017) 1-10.
[19] I. Grishchenko, M. Maffei, C. Schneidewind, A semantic framework for the securityanalysisofethereumsmartcontracts, in: L. Bauer, R. Ku"sters (Eds.), Principles of Security and Trust, Springer International Publishing, Cham, 2018, pp. 243-269.

[20] L. Jiang, How to identify a true blockchain use case with 11 questions (2018). https://hackernoon.com/breakingblockchain-a-framework-to-evaluate-blockchain-use-cases9efbc30a3fa7

[21] M. D. L. S. E. D. L. R. SCIENTIFIQUE, Indicateursstatistiquessur les dipl`om'es de l'enseignementsuperieur des secteurs public etpriv'e (2016). URL http://www.mes.tn/image.php?id=7635 